\title{
The DLR MIRO - A versatile lightweight robot for surgical applications
}

U. Hagn, M. Nickl, S. Jörg, G. Passig, T. Bahls, A. Nothhelfer, F. Hacker, L. Le-Tien, A. AlbuSchäffer, R. Konietschke, M. Grebenstein, R. Warpup, R. Haslinger, M. Frommberger, and G. Hirzinger

Institute of Robotics and Mechatronics, German Aerospace Center (DLR), Germany

E-mail: ulrich.hagn@dlr.de, mathias.nick1@dlr.de, stefan.joerg@dlr.de, georg.passig@dlr.de, thomas.bahls@dlr.de, alexander.nothhelfer@dlr.de,franz.hacker@dlr.de,luc.le-tien@dlr.de, alin.albu-schaeffer@dlr.de,rainer.konietschke@dlr.de,markus.grebenstein@dlr.de, rebecca.warpup@dlr.de, robert.haslinger@dlr.de, mirko.frommberger@dlr.de,gerd.hirzinger@dlr.de

\begin{abstract}
The paper presents a new torque-controlled lightweight robot for medical procedures developed at the Institute of Robotics and Mechatronics of the German Aerospace Center. Based on the experiences in lightweight robotics and anthropomorphic robotic hands, a small robot arm with 7 axis and torque-controlled joints tailored to surgical procedures has been designed. With an optimized anthropomorphic kinematics, integrated multi-modal sensors and flexible robot control architecture, the first prototype KINEMEDIC and the new generation MIRO, enhanced for endoscopic surgery, can easily be adapted to a wide range of different medical procedures and scenarios by the use of specialized instruments and compiling workflows within the robot control. With the options of both, Cartesian impedance and position control, MIRO is suited for tele-manipulation, shared autonomy and completely autonomous procedures. This paper focuses on system and hardware design of the robot, supplemented with a brief description on new specific control methods for the MIRO robot.
\end{abstract}

\section{INTRODUCTION}

Surgical robotic systems can be divided into two mayor groups: specialized and versatile systems. Specialized systems focus either on a dedicated surgical technique, like endoscopic surgery with the $d a$ Vinci surgical system by Intuitive Surgical (Green et al., 1995) or on the treatment of a specific medical disease (e.g. cancer in Phee et al., 2005). These systems can fulfil the dedicated task very good, but link the financial amortization in the clinic to single medical procedures. With ongoing research in medical treatment, many of these specialized robotic systems are likely to loose their niche. On the other hand, versatile systems today often still base on the adaptation of industrial robots (e.g. 
Caspar in Albers et al., 2007). Industrial robots are targeted on high accuracy and repeatability which is achieved by stiff structures and thus relatively high mass and large size. Space consumption, safety and adequacy in the unstructured and crowded environment of an operating room and in close human robot interaction are therefore at least questionable.

In contrast, the design approach of the DLR KINEMEDIC and the new generation MIRO aims at a compact, slim and lightweight robot arm as a versatile core component for various existing and future medical robotic procedures. With its low weight of $10 \mathrm{~kg}$ and dimensions similar to those of the human arm, the MIRO robot can assist the surgeon directly at the operating table without interference. Like the DLR Lightweight Robot (LWR in Albu-Schäffer et al., 2007), MIRO integrates torque sensing capabilities to enable close interaction with humans in unstructured environments. With the lightweight approach in the MIRO development, the accelerated masses are relatively low, which enhances the safety of the system during close interaction with patient and user (Haddadin et al., 2007). Compact and slim design simplifies the integration of one or multiple MIRO robots in the crowded operating room.

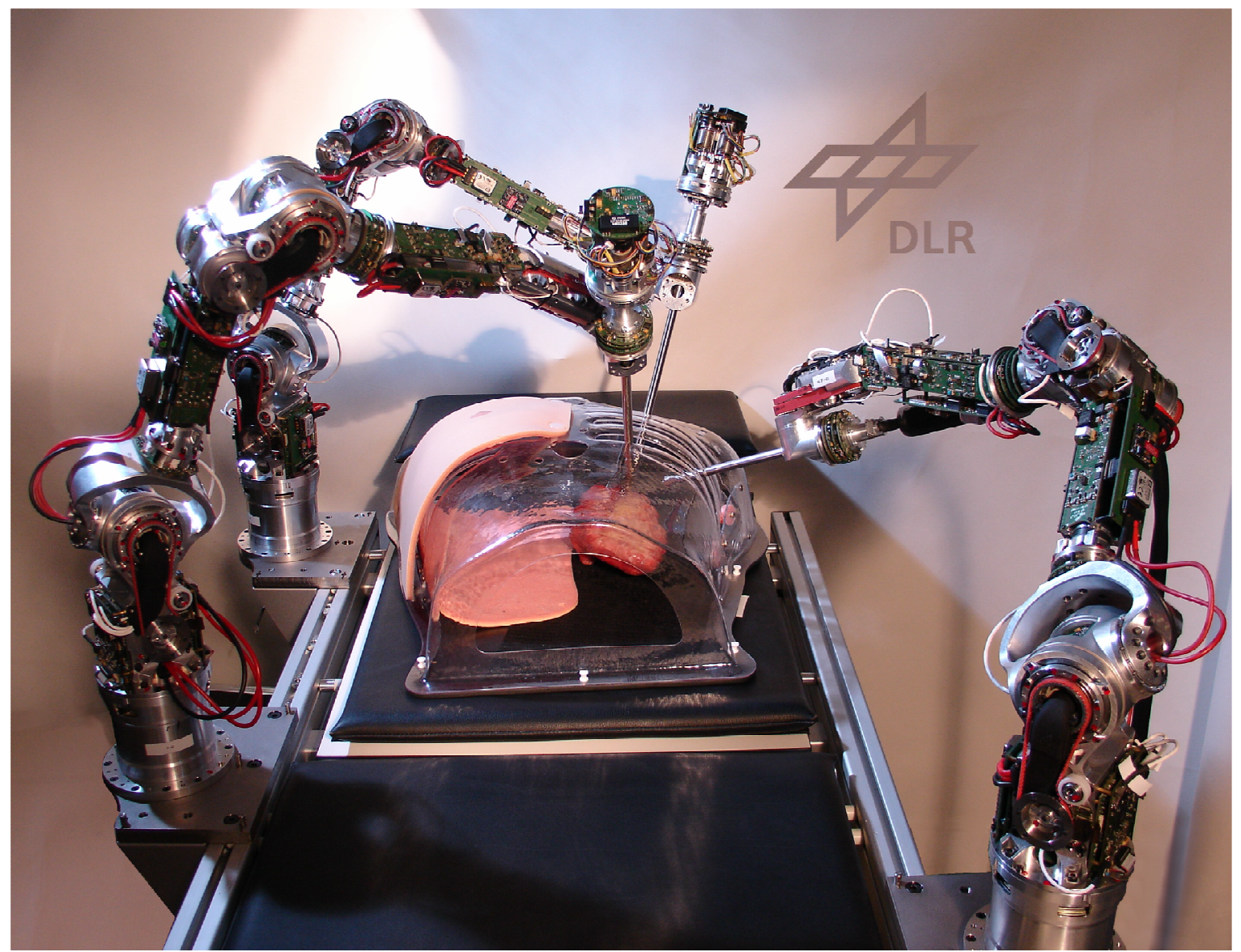

Figure 1: DLR MIRO robots equipped with endoscopic instruments in a tele-manipulation scenario (heart surgery) 
By adding specialized instruments and modification of the application workflows within the robot control, the MIRO robot can be adapted to many different surgical procedures. This versatility has been achieved by the design of the robotic arm itself and by the flexibility of the robot control architecture. To equalize the deficits of the lightweight approach in dynamic absolute accuracy, the MIRO robot control offers the possibility to integrate external position sensors to close the position control loop. In almost every surgical procedure where high absolute accuracy is needed, a procedure or/and an imaging systems for registration of the patient on pre-recorded diagnostic data are state-ofthe-art. These systems (e.g. navigation systems) can be used as external position sensors for the MIRO robot control to achieve a high absolute accuracy with the robotic system. In the understanding of the MIRO developers, the four basic parts of a surgical robotic system can be described as:

- surgeon - medical know how, surveillance, flexibility, responsibility

- robot - exact relative positioning, endurance

- instruments - adaptation to a specific surgical task, dexterity

- navigation - exact acquisition and planning of absolute positions

Beside classic position control, the robot can be guided by the user through exerting forces by hand on the robot's structure, which is implemented by using integrated torque sensors in all joints and torque control methods. This control method is described in (Albu-Schäffer et al., 2007) as soft robotics. With the different control modes the following robotic control architectures are possible:

- One or more MIRO robots are tele-manipulated by the surgeon from a distant control interface for example in endoscopic surgery.

- The robot is equipped with an instrument (e.g. drilling machine) and is guided by the surgeon manually, whereas the robot limits the free motion based on pre-planned virtual spaces and directions in the form of haptic paths, targets, virtual walls.

- The robot is equipped with an instrument (e.g. $\mathrm{CO}_{2}$ laser for osteotomy) and performs a planned trajectory autonomously and is registered by additional sensors/ procedures.

Two exemplary soft robotics applications have been implemented already on the first generation the DLR KINEMEDIC, which is in the commercialization phase at BrainLAB / Germany. Integrated into BrainLAB's surgical navigation system, the KINEMEDIC prototype performs biopsies in cranial neurosurgery and drills holes for pedicle screws in spine stabilization (Ortmaier et al., 2006). In both 
applications, the robot guides the surgeon to the point of interest (tumour or bore axis) by haptic means, whereas in the final step the robot simply holds the correct position and the surgeon completes the task manually.

Additionally, different setups in the operating room can be combined with these control architectures. Due to the lightweight approach, programmable gravity compensation and the corresponding dimensioning of the robot's joints, the MIRO robot can be mounted hanging from a ceiling stand (Frumento et al., 2006), on a mobile cart or at the side rails of the operating table, as shown in Fig. 1. The assignment of control modes, number of robots and the different setups is interchangeable.

\section{MIRO SYSTEM DESIGN}

Beside the lightweight approach, one essential design criterion of the MIRO robot is the versatility of the system. It is designed to fit seamlessly into existing surgical procedures and clinical environments, but is also configurable and extendable to comply with rapidly changing development in medical treatment and safety. To achieve this challenging demand, the MIRO robot has been conceived by clearly distinguishing between platform and application according to the principles of platform-based design (Sangiovanni-Vincentelli et al., 2004). In this context, the application is designated to configure and parameterize the platform for a specific task. To achieve the demanded versatility of the system, it is necessary to cover as many system constraints as possible by the configuration, due to the faster optimization cycles in application design. Only absolute necessary, static system constraints which are inherent to the field of use (surgery) or unlikely to change have been assigned to the platform. All remaining constraints are classified as open and can be realized within the application. In order to avoid limitations in the number of valid applications, the MIRO platform design encapsulates all fundamental functions of the system into small, decoupled and tested blocks. The communication infrastructure of the MIRO platform grants low-level, direct and independent but yet convenient access to these blocks and is open for future extensions of the platform. With this, application design can start directly on the hardware level and is only limited by the set of basic functions of the platform. The extent and impact of this design approach in the MIRO robot can be illustrated by some of the assignments of open and static constraints to application and platform, as shown in table 1.

The MIRO platform enables motion of the TCP in 6 degrees of freedom, restrictions deriving from the surgical procedure can be considered as being part of application. In endoscopic surgery, 
instruments are inserted into the patient's body through small incisions or orifices, which limit the motion of the instruments to 4 degrees of freedom. Different platform-based solutions have been introduced to restrict the instrument motions according to this limitation either by specialized mechanisms (e.g. daVinci system) or by additional passive joints (e.g. Aesop, Zeus). In contrast, the MIRO robot offers the possibility to solve this problem within the application, either by Cartesian position or impedance control about a virtual point of entry, which is assumed stationary or detected by sensors, or by two joints of the robot that are controlled to output zero torque.

\begin{tabular}{|c|c|c|c|}
\hline constraint & description & platform & application \\
\hline cartesian kinematics & unlimited motion of the TCP in 6 degrees of freedom & ○ & \\
\hline application kinematics & $\begin{array}{l}\text { motion restrictions specific to a certain surgical procedure } \\
\text { (e.g. motion about the invariant trocar in endoscopy) }\end{array}$ & & O \\
\hline relative accuracy & minimal size of anatomic structures (e.g. in neurosurgery) & ○ & \\
\hline absolute accuracy & $\begin{array}{l}\text { combination of relative accuracy with registration, } \\
\text { additional sensors and model calculation }\end{array}$ & & O \\
\hline $\begin{array}{l}\text { sensory measurement of } \\
\text { joint torques and positions }\end{array}$ & $\begin{array}{l}\text { integrated joint position and torque sensors are capable of } \\
\text { oversampling }\end{array}$ & o & \\
\hline $\begin{array}{l}\text { sensor sample rate and } \\
\text { resolution }\end{array}$ & $\begin{array}{l}\text { task specific adjustment of the ratio between sensor sample } \\
\text { rate and resolution }\end{array}$ & & o \\
\hline $\begin{array}{l}\text { space limitations in the } \\
\text { operating room }\end{array}$ & slim and compact design of the robot arm & o & \\
\hline $\begin{array}{l}\text { diversity of operating } \\
\text { rooms }\end{array}$ & $\begin{array}{l}\text { omnidirectional placement of the robots base to enable } \\
\text { different setups }\end{array}$ & & O \\
\hline basic safety functions & independent basic safety functions (e.g. redundancy) & $\circ$ & \\
\hline safety strategies & $\begin{array}{l}\text { combination of basic safety functions to comply with } \\
\text { different standards and applications }\end{array}$ & & O \\
\hline
\end{tabular}

Table 1: assignment of constraints to platform and application in the MIRO robot

The last two entities in table 1 partitions the safety of the system into basic safety functions of the platform (sensory and communication redundancy, safe stop function, etc.) and the combination of these functions by the application. This approach is necessary due to the different existing and oncoming safety standards in the field of medical robotics.

By methods of simulation (Konietschke et al., 2004), cadaver testing (Ortmaier et al., 2006) and interrogation of clinicians, sets of system requirements for a variety of challenging medical applications were identified. The range of applications includes endoscopic heart surgery, drilling for pedicle screws in spine stabilization, biopsy of tumours in neurosurgery and osteotomy with $\mathrm{CO}_{2}$ 
lasers in maxillofacial surgery. After partitioning these constraints either as static for the platform design or as open realized by the application, a set of design criteria have be formulated for the MIRO robot.

- slim, compact design to reduce the fear of close contact and to simplify the integration into the operating room

- lightweight to reduce the impact of collisions and to facilitate the setup in the operating room

- redundant number of joints to enhance flexibility and collision avoidance

- integrated sensors to measure all relevant physical properties of the system

- different control modes where the user can always be in charge

- integrated electronics to reduce the system's size

- pc-based external robot control for scalability reasons

- re-configurable electronics and robot control for rapid application prototyping

- open communication concept to integrate external sensors and actuators

\section{HARDWARE DESIGN}

\section{III.1. Kinematic Design}

The issue of acceptance of new technologies is important for an improved learning curve of clinicians and technicians. Close interaction with technical systems demand understanding of the system, thus a central design issue of the MIRO robot is an inherent predictability of the system's actions for the user. To achieve this, a serial kinematics with seven degrees of freedom which resembles those of the human arm has been developed and optimized for medical procedures. The joint morphology groups the MIRO arm in a dedicated shoulder (roll-pitch-yaw), upper arm, elbow (pitchroll), forearm and wrist (pitch-roll), each group with intersecting axes, as shown in Fig. 2. By this, the motions of the robot joints in relation to the Cartesian movement of the TCP are intuitive for the user, because they resemble those of the human arm. Only the wrist pitch-roll kinematics differs from the human pitch-yaw configuration, in respect of the frequently used rotation of endoscopic instruments about their shaft axis. The chosen kinematics offers 6 degrees of freedom at the TCP, additional restrictions deriving from certain medical procedures (e.g. in endoscopic surgery) can thereby be solved by the application. This almost anthropomorphic kinematics with dedicated groups of joints with intersecting axes and the slim design are the main differences in appearance between the MIRO robot and the DLR light weight robot (LWR). 
Based on the different selected medical procedures the kinematics of the robot has been optimized by the means of gradient methods and genetic algorithms (Konietschke et al., 2004). Beside conventional surgical procedures, the optimization process paid special attention to endoscopic procedures, due to the limitations in endoscopic surgery described in chapter II. After defining quality criteria such as position accuracy, manipulability, minimum dynamics, maximum force, etc. the joint angle ranges and the ratio between the length of the upper arm $\left(a_{3}\right)$ and the forearm $\left(d_{5}\right)$ have been optimized with the goal of a short overall length of the robot. The Denavit-Hartenberg parameters of the optimized kinematics are shown in table 2 according to the notation of Yoshikawa (Yoshikawa, 1990) and Craig (Craig, 1986).

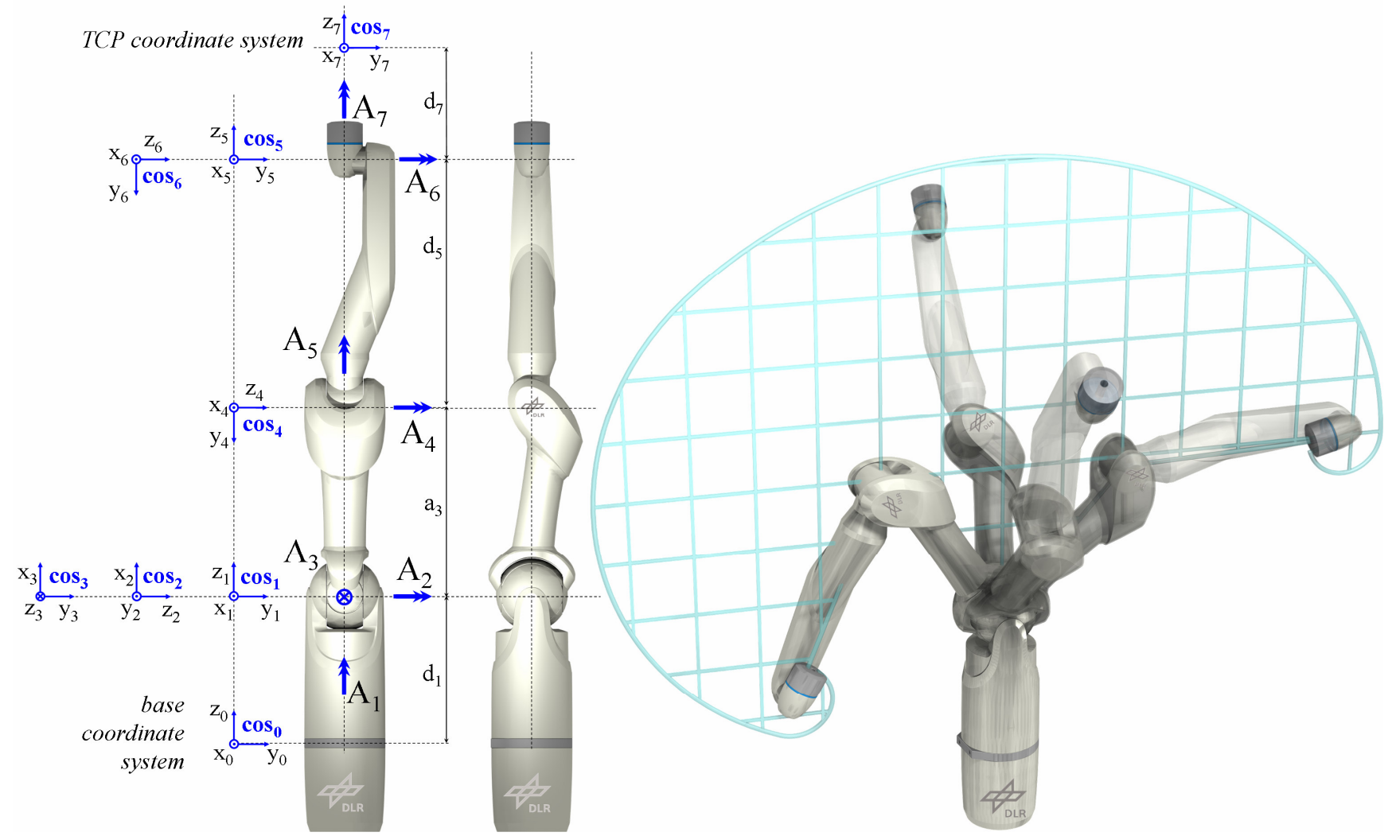

Figure 2: MIRO Kinematics (left); overlay of extremal pitch joint configurations with plot of maximum reach (right)

\begin{tabular}{|l|r|r|r|r|}
\hline$i$ & $a_{i-1}[\mathrm{~mm}]$ & $\alpha_{i-1}\left[{ }^{\circ}\right]$ & $d_{i}[\mathrm{~mm}]$ & $\left.\Theta_{0} \Gamma^{\circ}\right]$ \\
\hline 1 & 0 & 0 & 240 & 0 \\
\hline 2 & 0 & -90 & 0 & -90 \\
\hline 3 & 310 & 90 & 0 & 0 \\
\hline 4 & 0 & -90 & 0 & 90 \\
\hline 5 & 0 & 90 & 385 & 0 \\
\hline 6 & 0 & -90 & 0 & 0 \\
\hline 7 & 0 & 90 & 200 & 0 \\
\hline
\end{tabular}

\begin{tabular}{|l|r|r|}
\hline joint & range $\left[{ }^{\circ}\right]$ & software limit $\left[{ }^{\circ}\right]$ \\
\hline 1 & \pm 172.5 & \pm 162.5 \\
\hline 2 & \pm 51 & \pm 41 \\
\hline 3 & \pm 51 & \pm 41 \\
\hline 4 & $-57 /+127$ & $-47 /+117$ \\
\hline 5 & \pm 172.5 & \pm 162.5 \\
\hline 6 & \pm 172.5 & \pm 162.5 \\
\hline 7 & \pm 172.5 & \pm 162.5 \\
\hline
\end{tabular}

Table 2: DH parameters and joint ranges of the MIRO robot 


\section{III.2. Mechanical Design}

The integration of all hardware features established by the DLR LWR (torque and position sensors, safety brakes, integrated electronics, etc.) in a yet more slim design with dedicated groups of joints with intersecting axes is the most challenging aspect in the mechanical design of the MIRO robot. The design approach of the LWR, with a sequential chain of revolving single joints connected by shell structures (Hirzinger et al., 2002) turned out to be not feasible for the MIRO robot. In respect of good accessibility to the integrated electronics and the upper arm and forearm being the only places to integrate the electronics, this approach is not scalable to the desired slim dimensions of the MIRO robot. The mechanical design is therefore based on a skeletal structure and unencumbered housings. Similar to the bones in the human anatomy, these structural parts have been designed as tubes whereas the joint electronics are fitted to the outside. The decoupling of structural parts and housings gives the opportunity to design compliant polymer housings in order to reduce the severity of collisions, a measure which can also be found in automotive cockpit design. Additionally, the robot can be operated without housings as shown in Fig. 1 during testing and maintenance.

To form compact groups of joints with intersecting axes, couple joints with 2 DoF have been designed in analogy to the joints in the DLR Hand II (Butterfaß et al., 2001). The couple joint design offers various advantages:

- both motors / brakes on the same side of the double joint (bundling of electronic components)

- compact joint dimensions

- intersecting axes

- efficiency

The efficiency advantage needs further annotations. With the MIRO 2 DoF couple joint design the torques of the motors add for one degree of freedom under certain circumstances. If one joint torque is zero, the second joint torque is formed by the sum of both motor torques. As with the human arm, maximum payload needs not to be applicable in every kinematic posture. It is reasonable to use adequate kinematic postures of the robot arm in high payload procedures. In a robot with a redundant number of joints, Cartesian tasks of the TCP can be realized by various kinematic postures in the null space (Grunwald et al., 2004). The setup and posture of the robot can therefore be optimized to achieve certain advantages. In the case of the MIRO robot, a posture that creates mostly single axis loads on the couple joints offers the opportunity that the motor torques add as described above. 
The robot's shoulder is formed by a single revolving joint and a 2 DoF couple joint with intersecting axes (see Fig. 3). Joint 1 is designed similarly to the joints in the DLR LWR; motor and brake (M/B) are connected to a reduction gear (RG), a torque sensor (TS) and a position sensor (PS) on the joint side and position sensors on motor (not depicted) side are integrated. All joints in the MIRO robot feature a hollow shaft, which enables internal cabling of the robots electronics. The pitch and yaw degrees of freedom (axis 2 and 3) are realized by said couple joint design. Two motors equipped with safety brakes (M/B) are connected to reduction gears (RG) by tooth belts (TB). The two reduction gears are coupled by a Cardanic differential bevel gear (BG), whereas two bevels are connected to the drive side of the reduction gears, one bevel is running free (optional) and the driven bevel is connected to link 3. When both motors rotate in the same direction, the couple joint rotates about axis 2, whereas opposite rotations of the motors result in a joint rotation about axis 3 (yaw). Because of the limited space in the joint due to the integration of the differential bevel gear, the torque sensor can not be integrated in the way used in joint 1 . Both torques of the couple joint are measured by a two axis torque sensor which is integrated into link 3. The joint position sensors (PS) of all joints are integrated in a way which is not affected by the coupling mechanism and therefore measure directly the joint positions.

To form a pitch roll configuration for the elbow of the MIRO robot, a variation of the couple joint is used. The index position of the cardanic differential bevel gear (BG) is rotated by 90 degree about axis 4 and link 5 is connected to the driven bevel as shown in Fig. 3. The torque sensor (TS) measures the torque about axis 5 and two additional orthogonal torques. Based on the known orientation of axis 5, the torque about axis 4 can be calculated out of these two additional signals.
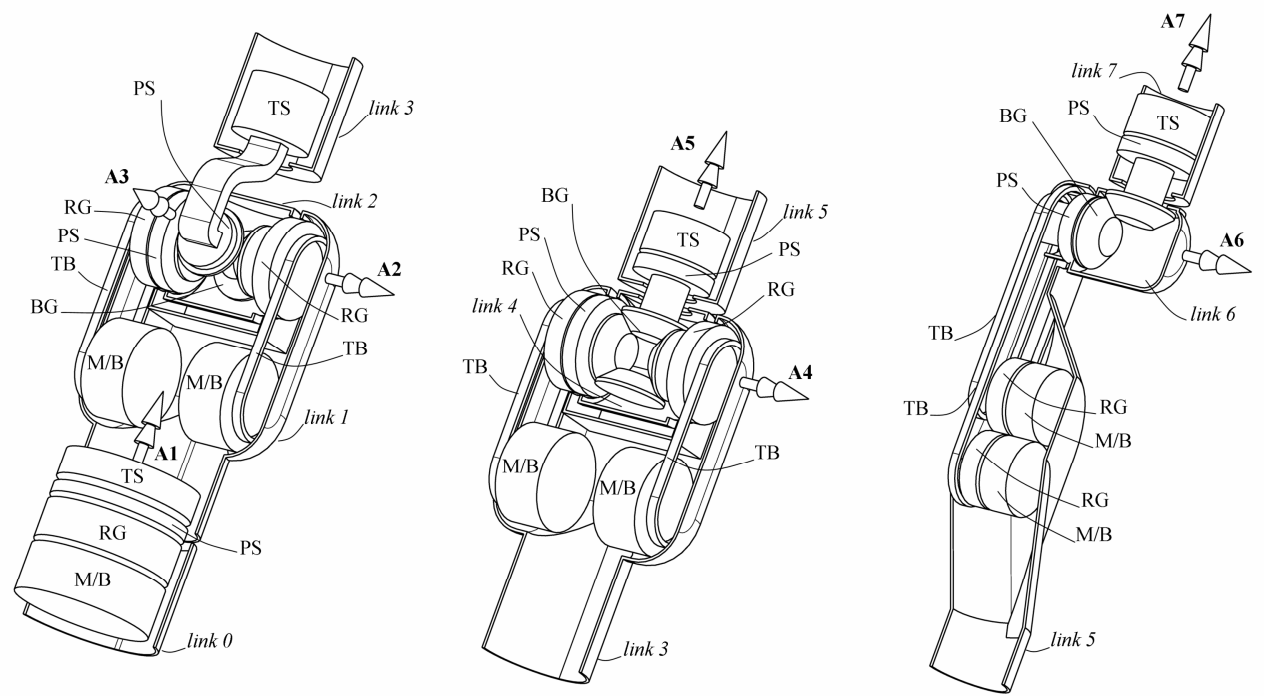

Figure 3: Shoulder joints 1, 2, 3 (left), elbow joints 4, 5 (middle) and wrist joints 6, 7 (right) of the MIRO robot 
In order to achieve a very compact pitch-roll wrist a different coupling mechanism has been designed for joints 6 and 7, which does not offer the above mentioned efficiency advantage. Two modules containing motor, break (M/B) and reduction gear are connected to the joint by tooth belts (TB), as shown in Fig. 3. One tooth belt directly drives link 6, whereas the second belt is connected to the driving bevel of a differential gear assembly (BG). The driven bevel is connected to link 7 and the torque sensor (TS), whereas the measurement of the torques is realized in the same way as in joint 4 and 5. Additionally, this joint design offers large joint ranges for axis 6 and 7 which is necessary for the use with endoscopic instruments.

Endoscopic instruments can be portioned into the functional tip (gripper, scissor, optics, etc.), a long thin shaft and an extracorporeal supply unit (see Fig. 4). For safety reasons it is important that the instrument can be removed from the patients body along the axis of the shaft. Whereas conventional instruments (e.g. drill machines, saws, lasers), can be mounted to the MIRO as shown in Fig 4 left, the hollow shaft of axis 7 and the large range of axis $6\left( \pm 162.5^{\circ}\right)$ allows endoscopic instruments to be mounted as depicted in Fig. 4 (right). An endoscopic instrument (e.g. DLR's actuated and sensorized surgical instruments in Seibold et al., 2005) can be inserted through the hollow shaft of joint 7 and points with its functional tip in the opposite direction of the $\cos _{7}$ coordinate system's z-axis. The rotational degree of freedom about the axis of the shaft has been identified as very frequently used during endoscopic surgery and is therefore realized by a dedicated joint of the robot in order to reduce expansive robot motions.
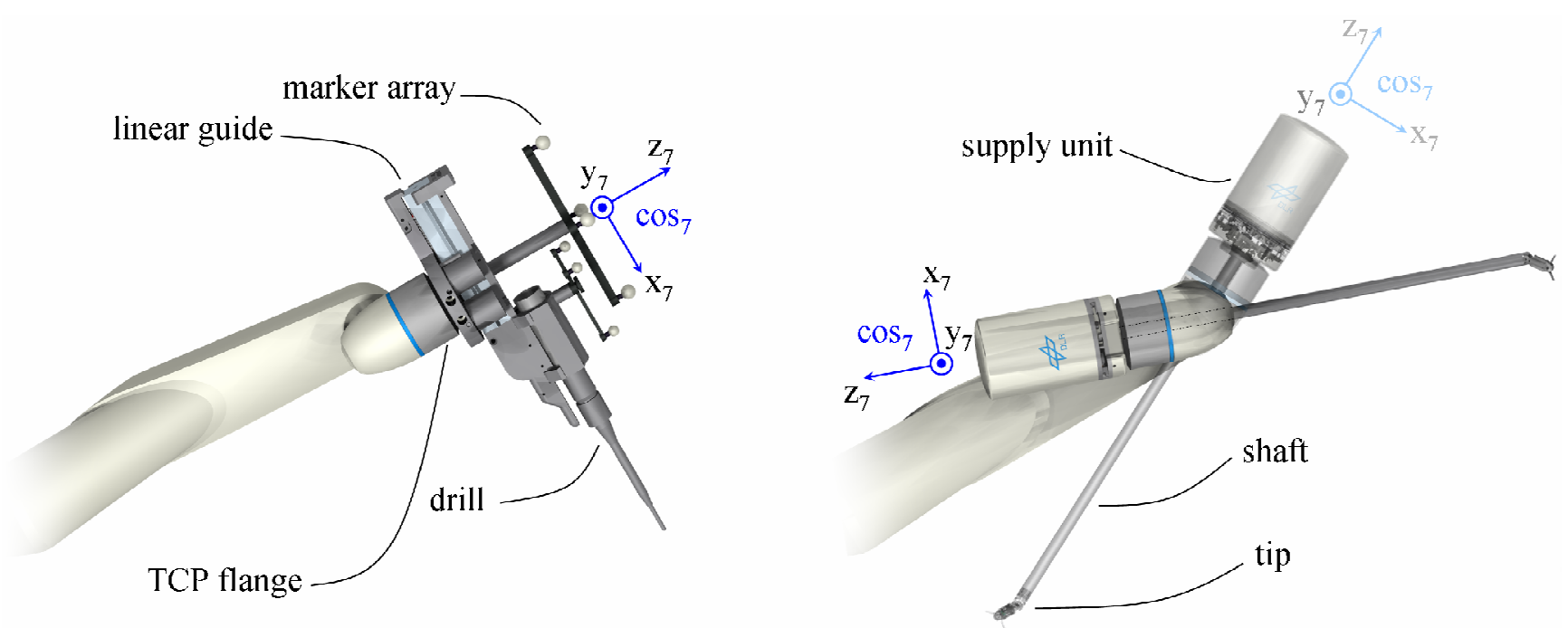

Figure 4: MIRO robot equipped with passive linear guide and a drill machine (left); with endoscopic instrument in two extremal positions (right). 


\section{III.3. Electronic Design}

From the viewpoint of electronic architecture the robotic system consists of sensors and actuators, the interfaces to the physical world, which are integrated with a heterogeneous communication infrastructure. The design of these sensor and actuator components must fit into the mechanical design and these components are hooked into a communication infrastructure that determines strict real-time and protocol constraints. So, the art of sensor and actuator design for high integrated robots is to find the best trade-off between a highly integrated and efficient implementation, to meet the strict constraints, and a suitable modularization, to simplify the design by reusing common components.

The modularisation comprehends communication protocols and configurable functionality. In contrast, the shapes of the modules are adapted individually regarding the morphology of the mechanical structure. So, the small footprint of the resulting modules enables a high level of integration. And in spite of sparse integration space, the modules offer a high degree of flexibility.

All these modules implement BiSS (IC-Haus, 2003), a unified sensor/actuator communication bus with very small footprint. The BiSS protocol allows cascading: different BiSS-components can be connected to a chain and only the first component of the chain is connected to the BiSS-Master, so that multiple sensor modules can be combined to a stack with a single BiSS-interface. Hence, BiSS allows a high degree of integration and the distribution of the modules according to the different joint designs.

A MIRO robot joints consits of three types of common components, which realize the interfaces to the physical world: torque sensing module (TSM), position sensing module (PSM) and actuation module (AM). Accordingly, a Miro joint is the results of a dedicated composition of sensing and actuation modules.

\begin{tabular}{|l|l|l|l|}
\hline & Actuation & Single Sensor Modules & Stacked Sensor Modules \\
\hline Joint1 & $1 \mathrm{AM}$ & & 1 PSM, 1 TSM \\
\hline Joint23 & $2 \mathrm{AM}$ & $2 \mathrm{PSM}$ & 1 TSM \\
\hline Joint45 & $2 \mathrm{AM}$ & 1 PSM & 1 PSM, 2 TSM \\
\hline Joint67 & $2 \mathrm{AM}$ & $1 \mathrm{PSM}$ & 1 PSM, 2 TSM \\
\hline
\end{tabular}

Table 3: Distribution of Sensor and Actuator modules in the MIRO robot

\section{III.3.1. Sensing Modules}

All electronic parts of the sensor modules (TSM and PSM) including A/D and BiSS interface implementation are located at the point of measurement, close to the strain gages or the potentiometers. Thus the torque sensing module and the position sensor module are self-contained 
units which only need a power supply and communicate via the standardized BiSS interface. They can be combined in different arrangements as needed in the different joints of the robot (see Fig. 3). All torque sensor modules and position sensor modules arranged in the same location can share the same power supply and the same BiSS interface. So the expense of cabling is minimized.

The angular positions of the robot's joints are measured through conductive plastic potentiometers. The linearity error of these potentiometers is trimmed to less than $0.1 \%$. The position sensor electronics acquires the resistive ratios of the potentiometers and converts them to digital values for further processing. The potentiometer voltage is buffered by an operational amplifier which also implements second order anti alias low pass filtering with a cut-off-frequency of $800 \mathrm{~Hz}$. The filtered analogue voltage is converted to a digital word by a 16bit SAR A/D converter.

The torque sensors in the joints of the robot are based on aluminium parts with applied strain gages. These sensor bodies are integral part of the robot's structure as described in section III.2. The joint side torques and forces cause an elastic deformation of the sensor bodies. This deformation leads to a change in resistance of the strain gages. The strain gages are distributed over the sensor body in a way that the effects of unwanted forces and torques are compensated in a bridge while the torque parallel to the joint axis causes a detuning of the bridge. Special integrated time-to-digital A/D converters are used to acquire the change in the resistive ratio of the single half bridges of strain gages. The measurement rate, resolution, offset, gain and filter parameters of these circuits are programmable in a wide range. Therefore no hardware alignment of the strain gage bridges is needed and the sensors can easily be adapted to different demands.

\section{III.3.2. Actuation Module}

The actuation module (AM) is a high integrated current control module for brushless DC motors with a standard serial communication interface and a simple DC Power interface (5V5 electronic supply and 48V motor supply).

As an interface to the physical world, this module should represent a linear torque source. Therefore, the servo unit is arranged around a Xilinx Spartan-3e XC3S1200 FPGA running a current controller with space vector modulation (Quang N., 1999). The three phase bridge of the power converter is designed to provide up to $10 \mathrm{~A}$ at $48 \mathrm{~V}$ with a footprint of only $42 \mathrm{~mm} \times 25 \mathrm{~mm} \times 4 \mathrm{~mm}$, including three current sensors. A magneto resistive position sensor (2304 increments per electrical round) allows current control with little phase error. The high PWM frequency of $100 \mathrm{kHz}$ and the use of low ESR ceramic capacitors reduce the overall filter capacitance and inductivity, i.e the size of discrete passive components is reduced. 
Precise current control requires precise motor current measurement. This can be done directly in the motor phases but high voltage variations while measuring low voltage differences reduce measurement precision or require considerable instrumentation effort. This can be overcome by measuring the motor current on the source side of the low-side MOSFETs during current flow at this point. The AM implements this method by accepting the disadvantages due to the short acquisition time, while the low side is conductive.

To release the magnetic safety brake the FPGA implements a simple current controller circuit, which realizes a free programmable current profile. A higher current ensure that the brake is releasing reliably, and a reduced current minimize power dissipation of the released brake.

\section{INFRASTRUCTURE DESIGN: HARDWARE INTEGRATION AND SOFTWARE DRIVERS}

Infrastructure of a robotic system enables the integration of the distributed electronic hardware, such as sensors and actuators, with control applications. Therefore, it has to provide convenient interfaces for application developers and constitute efficient but transparent glue between the distributed components of the robotic system. Thus, infrastructure involves the operation environment for sensors and actuators, communication protocols and software drivers.

Infrastructure design for the MIRO robot is determined by two opposite prerequisites: On the one hand infrastructure should be an open platform that can be configured for versatile applications. This asks for a flexible and configurable architecture that is rich in functionality. On the other hand the electronic components are to be highly integrated with the mechanical structure. Thus, infrastructure needs to be lean and efficient to honor the sparse resources.

To balance these opposing requirements, a hierarchical approach is used: Close to the mechanical structure, where available space is sparse, components are most dedicated. Higher layers successively add flexibility to the system leading to the use of general-purpose architectures on the top layer (see Fig. 5). 


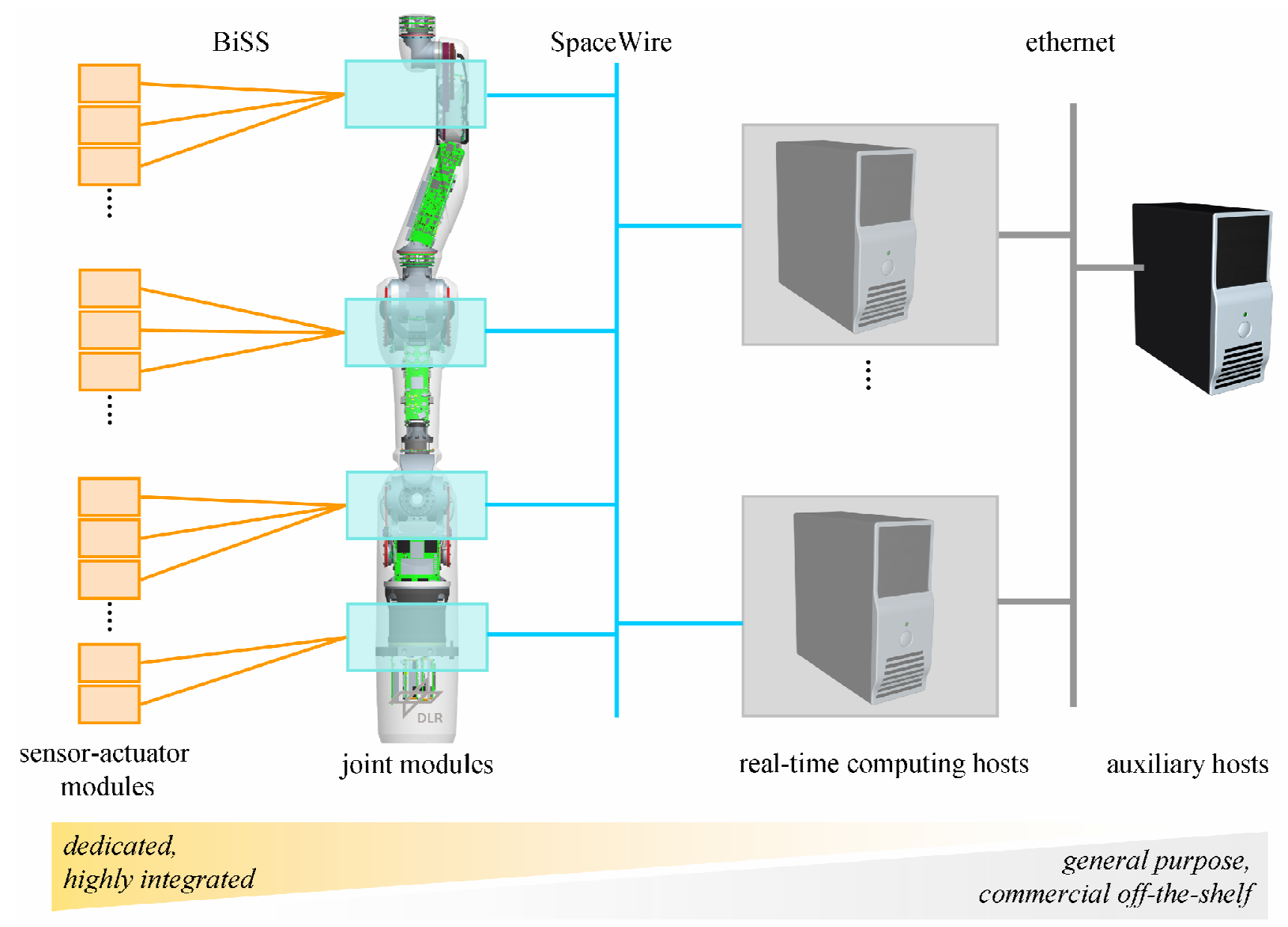

Figure 5: The hardware architecture features three layers of communication: BiSS, SpaceWire and Ethernet

The challenge for designers is the decision which functionality to implement on which layer. The dedicated components on the lower levels require high design effort and long development cycles. Hence, only functionality related to the platform should be implemented on lower levels. It is desirable to put most of the functionality on higher levels, but dedicated constraints such as high bandwidth or safety draw functionality to bottom layers.

While the classical system view is rather software-centric, the Miro-architecture is radically different: The entire system is synchronized by the sensors. The high-level software implementations of control algorithms are triggered by the joint hardware and the software is executed on demand, i.e. when new data are available.

In contrast to the classical hierarchy of hardware/firmware/device-drivers/software that separates hardware from software, the Miro-architecture is built on a component model. Components can be either hardware or software implementations. Components are the design entities on all levels of the system, be it electronic circuits or high-level software implementations. A component model is functionality driven not implementation driven like the device driver view. 
The synchronous signal-oriented design model introduced by Benveniste et al., 2003 is used to define the interfaces and the interaction of the distributed components. This formal definition allows the seamless composition of components on all levels of the system hierarchy, which in turn enables the flexible movement of functionality between layers, regardless of hardware or software implementation.

\section{IV.1. The Platform Architecture}

To balance the opposing requirements of flexibility and efficient implementation the MIRO platform is designed as a layered architecture, starting at dedicated, highly integrated components growing towards general-purpose, commercial off-the shelf platforms (see Fig 5).

The applied communication protocols follow the same hierarchy as the modules: Close to the hardware, low bandwidth protocols featuring small implementation footprint are used, while the general-purpose platforms are connected by high-bandwidth standard communication protocols.

The modular layout on each level together with the aggregation of components on successive levels by the means of suitable communication creates the desired platform flexibility. The four layers sensor/actuator modules, joint modules, real-time hosts, and auxiliary hosts have proven to be a suitable hierarchy with sufficient flexibility at affordable design costs for robotic systems.

The sensor-actuator modules constitute the most dedicated level of the architecture as they represent the interface to the physical world (see Sec. III). The next level is formed by the MIRO joint modules, which are the main building blocks of the robotic system. They provide the infrastructure for the integration of the various sensor and actuator modules. Hence, the key for an open platform is the flexibility of the joint modules and the communication connecting them.

At this granularity, Field Programmable Gate Array (FPGA) technology is a suitable means for providing the desired flexibility at reasonable design costs. For example, the great number of available I/O pins of the FPGA allows the parallel connection of all sensor/actuator modules. Thus, low bandwidth communication protocols featuring small implementation footprints can be used here. The sensor-actuator modules are connected to the joint modules with the industry standard sensor bus BiSS in C-Mode version.

The MIRO joint module is an instantiation of the flexible joint module concept described in Jörg et al., 2006. It uses a XILINX Spartan 3E FPGA for the implementation of the connection to the local modules (see Fig. 6). Joint housekeeping and state monitoring are also implemented on the FPGA. 
The joint modules are connected to real-time computing hosts by SpaceWire featuring $1 \mathrm{GBit} / \mathrm{sec}$ bandwidth and a latency lower than $20 \mu$ s (ECCS, 1998). The packet based SpaceWire offers a flexible and ample communication. Its protocol is entirely implemented on the joint FPGAs.

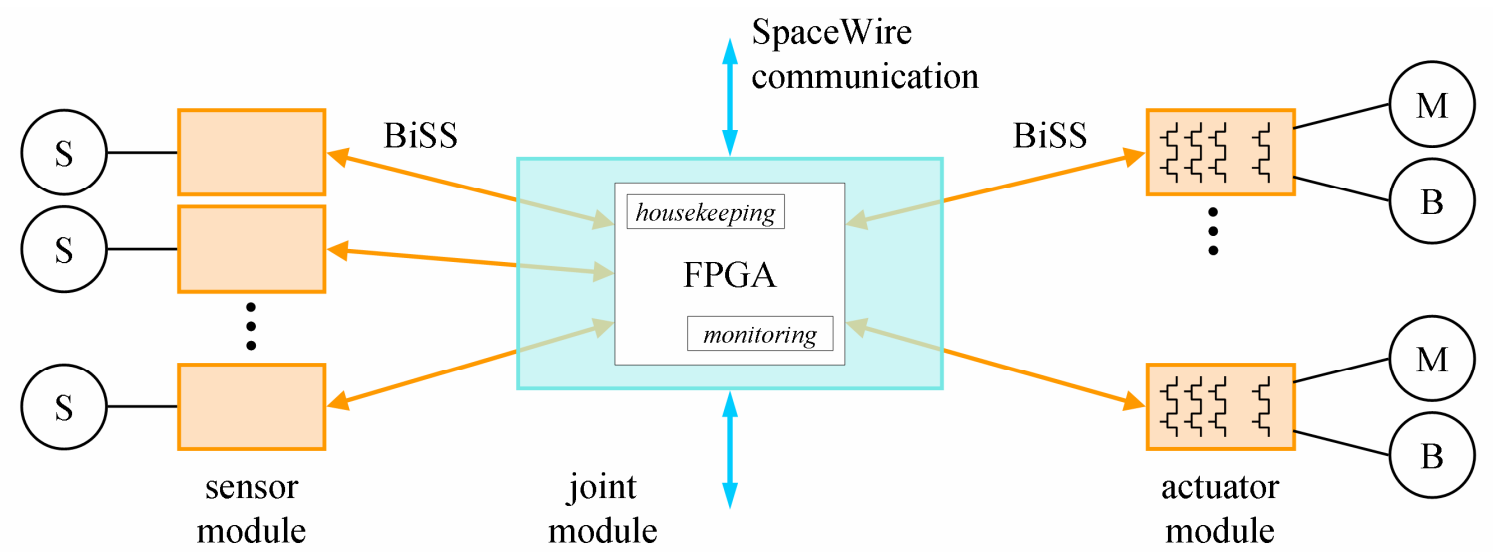

Figure 6: MIRO Joint Module: An open platform that allows the easy adaptation to changing application and safety requirements

Real-time computing hosts provide the necessary computing power for the control implementation. The top level is the interconnection of real-time computing hosts to auxiliary hosts via standard Gigabit Ethernet. For example, the hosts of a multi-arm robotic setup as pictured in Fig. 1 can be connected to one system in this manner.

\section{IV.2. The System Architecture}

Following the strategy to implement as much functionality as possible on general-purpose architectures all control algorithms are implemented on the external controller host, which is a standard PC platform running the real-time OS $\mathrm{QNX}^{1}$. This approach allows the use of conventional development tools such as Mathwork's Matlab Simulink and enables efficient algorithm and application development. Moreover, one directly benefits from the constantly rising computing power of Commercial of the Shelf (COTS) platforms.

To achieve good performance in terms of control a tight integration of the joint modules with the host by the infrastructure is required. In conformance to the signal model the distributed joint module functionality is transparently integrated by a signal-flow-oriented middleware specifically developed for this purpose. Utilizing a static system specification approach and compile time optimization the

\footnotetext{
${ }^{1}$ QNX Software Systems, http://www.qnx.com
} 
typical overhead of common middleware implementations is avoided and a small run-time footprint is achieved (Jörg, et al., 2006). The middleware not only integrates the hardware with the software but enables hardware-software co-design. It covers one important common task: the communication.

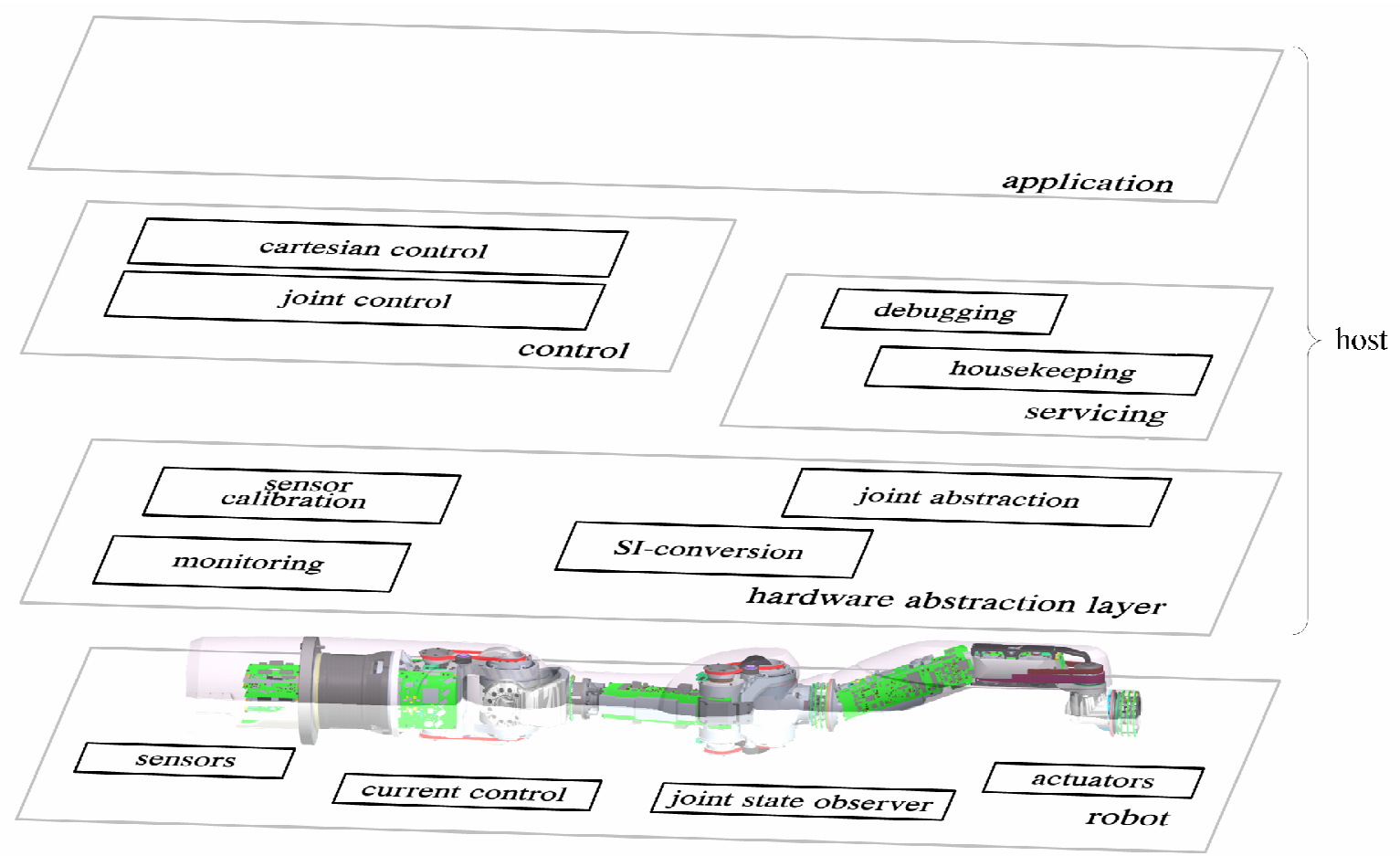

Figure 7: The layered MIRO system architecture: Only the current control is implemented on the joint modules. The Hardware Abstraction Layer (HAL) provides the interface to Control and Servicing.

Figure 7 depicts the system architecture of the MIRO system: The medical application configures the joint control and the Cartesian control laws of the Control Layer according to its requirements. Sec. $\mathrm{V}$ explains this procedure in more detail.

The Hardware Abstraction Layer (HAL) provides a functional separation between robot hardware and robot control. For convenience, the HAL implementation provides a complete current controlled robot to the control designers and presents all sensor values as floating point SI-values. Since the Cartesian control and the joint control are implemented with Matlab Simulink the HAL is represented as a Simulink block. The HAL not only integrates the remote joint functionality but also implements all features that are not provided by the hardware but are necessary for a suitable view of a current controlled robot, such as sensor value calibration (see Fig. 7). 
Only the current control as well as dedicated platform specific functions are implemented on the joint modules or the actuator and sensor modules, due to its high bandwidth or safety requirements.

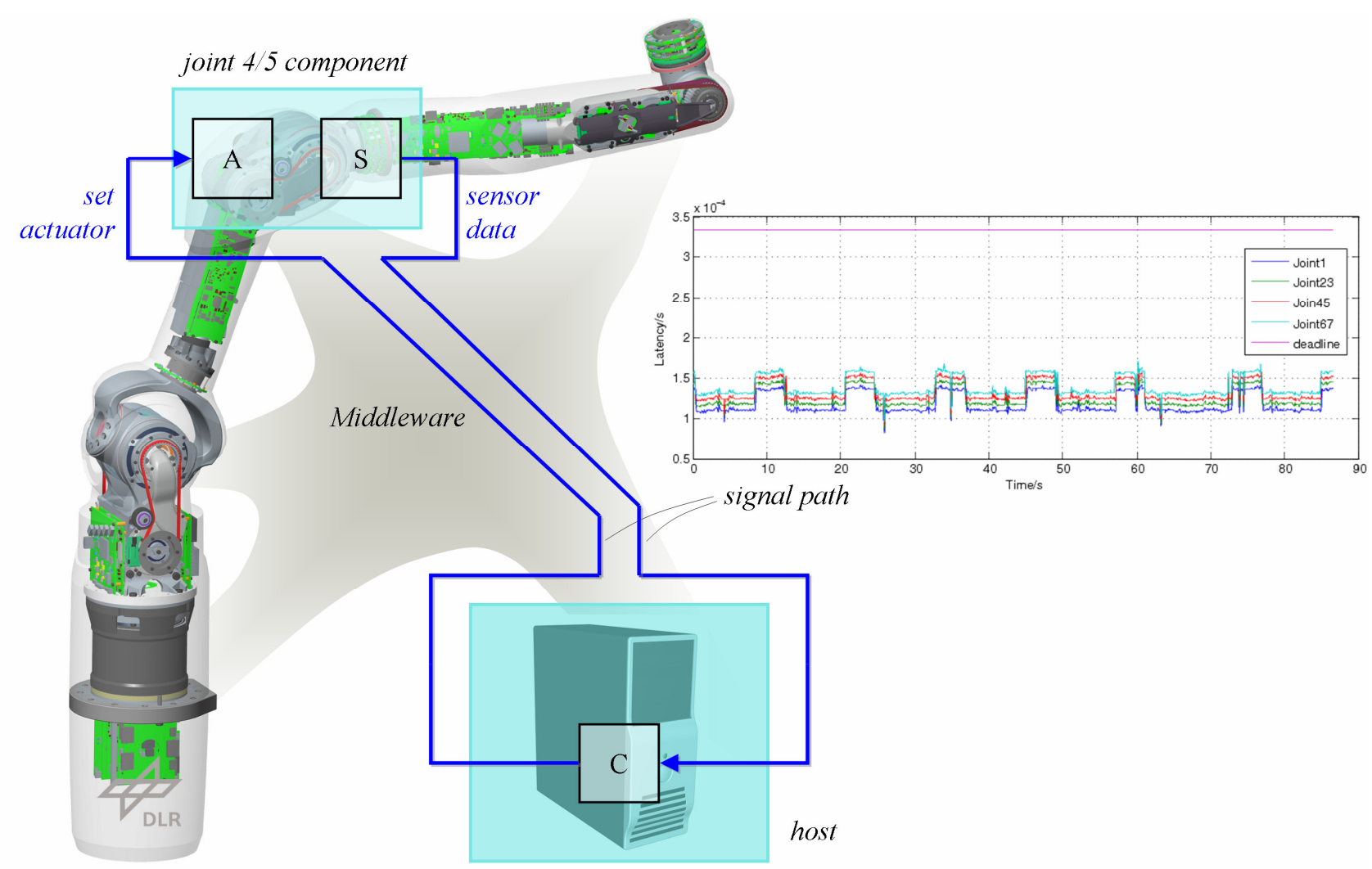

Figure 8: From the signal path to the middleware view: The signal path mapped to the robot hardware yields signaloriented components (i.e. the joint modules). The plot depicts the typical latency of the entire path.

Efficiency in terms of control design can be expressed by bandwidth and latency. The $1 \mathrm{GBit} / \mathrm{s}$ bandwidth of the SpaceWire compared to typical data of 250 byte/cycle allow theoretical control cycles of more then $500 \mathrm{kHz}$, which is several times more than the bandwidth of typical physical processes. Fig. 8 shows the overall latency from sensor to actuator that was measured while the robot was performing a typical position controlled trajectory. One can see that the latency always stays well bellow the deadline of one joint control cycle (i.e. $333 \mu$ s).

\section{ROBOT CONTROL}

The MIRO robot has two main control modes, which can be selected by the application (see Fig 9). The first one is the classical position control mode, in which the robot follows the commanded trajectory in Cartesian or joint coordinates as accurately as possible and is controlled to overcome 
external disturbances. This mode is required for exact positioning applications, such as laser cutting or bone drilling. The second operation mode is the compliant one, i.e. the so called soft robotics approach, briefly described in the following section.

\section{V.1. Torque and Impedance Control}

In this mode, the user can guide the robot to a desired position or on a desired trajectory by hand, in a highly intuitive manner (Albu-Schäffer et al., 2007). This is done in the so called "gravity compensation mode", in which a torque controller on joint level reduces the effects of friction and provides the torques needed to sustain the own weight of the robot and the tool. In this free-floating mode, several virtual springs based on virtual potential fields are used to impose constraint forces which prevent the robot entering restricted areas. This helps avoiding collisions with the patient, the surgeon and medical staff or with other instruments. Critical areas defined by the application (e.g. blood vessels or the spinal cord) are also avoided by the robot using this method. Moreover, by defining different compliances for the various Cartesian displacements of the robot, the system can accommodate to motion in free space (high stiffness) and contact with hard environments (requiring high compliance). This enables fast and sensitive interaction with the environment. Since the robot is a redundant arm with seven degrees of freedom, it can still be freely moved in the nullspace while having a Cartesian compliance imposed (Ott et al., 2004). In order to control also the nullspace configuration of the arm, additional null-space stiffness is defined. 


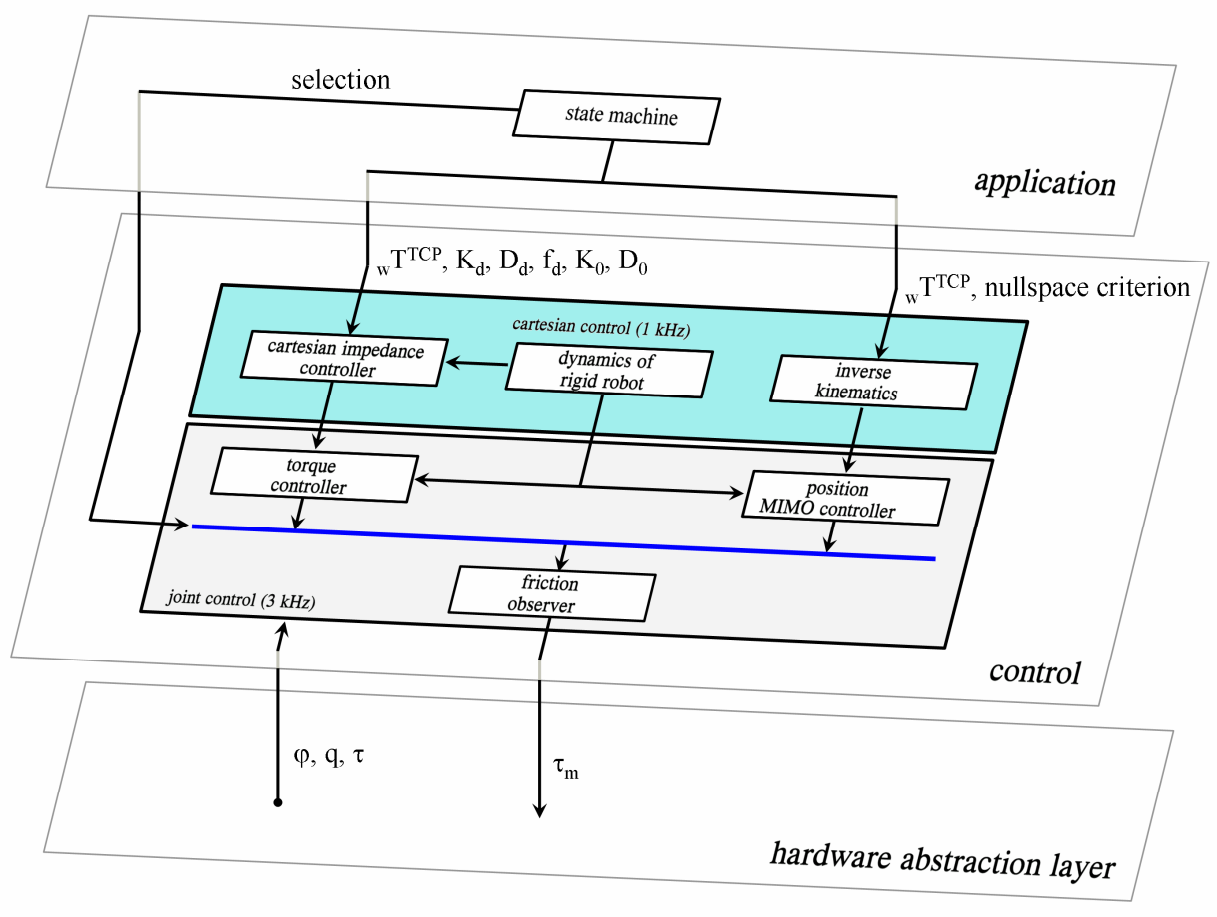

Figure 9: The controller structure for the DLR MIRO, whereas $\varphi$ is the link position, $\mathrm{q}$ the motor position, ${ }_{\mathrm{w}} \mathrm{T}^{\mathrm{TCP}}$ the desired Cartesian configuration, $\mathrm{K}_{\mathrm{d}}$ and $\mathrm{D}_{\mathrm{d}}$ the desired Cartesian stiffness and damping matrices, $\mathrm{f}_{\mathrm{d}}$ the desired TCP force vector, $\mathrm{K}_{0}$ and $\mathrm{D}_{0}$ the desired null-space stiffness and damping matrices.

\section{V.2. Position Control}

The main challenge regarding the position control is related to the fact that the robot exhibits significant elasticity due to the lightweight design. A flexible joint model has to be assumed therefore and the controller structure has to provide effective vibration damping and positioning accuracy in presence of elasticity. This is achieved by using a state feedback controller, with the states given by motor position and velocity as well as joint torque (after the gearbox) and its derivative $\{q, \dot{q}, \tau, \dot{\tau}\}$ (LeTien et al., 2007). This topic was addressed for the DLR lightweight robots as well. However, due to the special design of MIRO robots, the joints 2-3, 4-5, and 6-7 exhibit a strong coupling not only of the motion, but also of the elasticity and damping matrix. Therefore, the joint controllers cannot be designed separately any more, but the coupling of the two joints has to be considered, leading to a multi input- multi output (MIMO) design (see Fig. 10). 

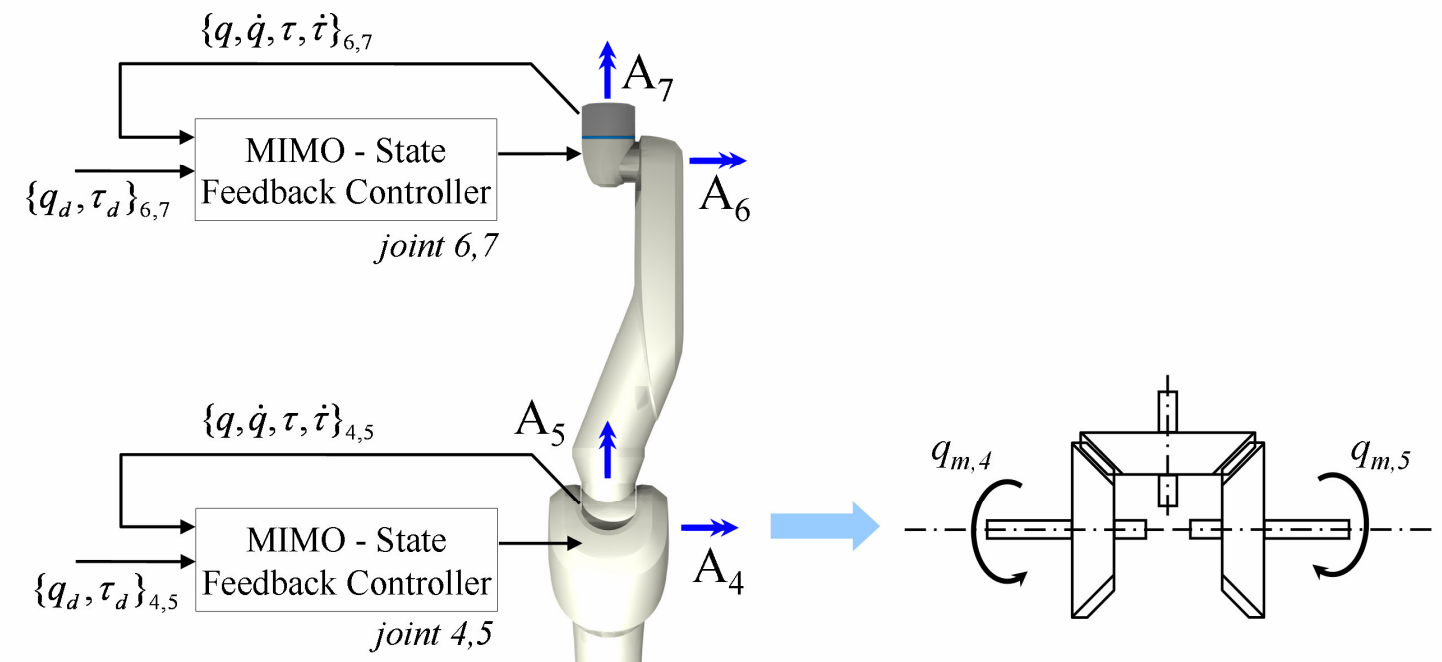

$\mathrm{A}_{3} \quad\{q, \dot{q}, \tau, \dot{\tau}\}_{2,3}$

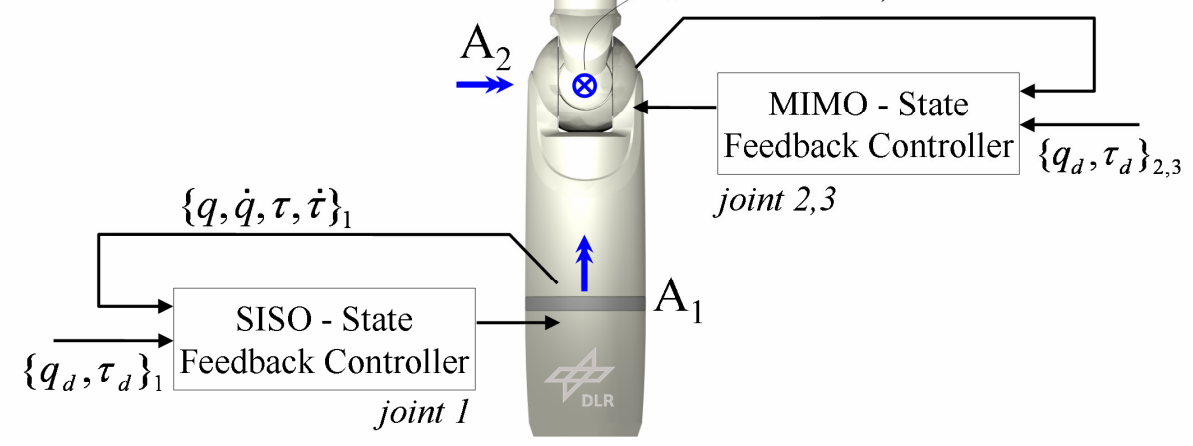

Figure 10: The structure of the MIMO state feedback controller for the coupled joints.

For the coupled joints, one has to distinguish between the motor positions measured by the encoders and the motor positions written in link coordinates. Accordingly, a coordinate transformation has to be done for transforming link side torques of the joints to motor torques. The two coordinate transformations are given by

$$
\left\{\begin{array}{l}
q=T q_{m} \\
\tau_{m}=T^{T} \tau
\end{array} \quad \text { with } \quad T=\left[\begin{array}{cc}
0.5 & 0.5 \\
-0.5 & 0.5
\end{array}\right] \in R^{2 \times 2}\right.
$$

Herein, $q_{m} \in R^{2}$ is the motor position, $\tau_{m} \in R^{2}$ the motor torque, $q \in R^{2}$ the motor position transformed to link coordinates and $\tau \in R^{2}$ the link torque. The controller design starts from the model written in joint coordinates on which a second coordinate transformation, using modal analysis is done for the coupled joints in order to attain optimal performance.

The feedback terms turn out to have very intuitive physical interpretations: torque feedback reduces the apparent inertia of the motors, as well as the joint friction. Motor position feedback is equivalent to a physical spring while velocity feedback produces energy dissipation (viscous friction). These 
interpretations allow a straight forward passivity and stability analysis based on Lyapunov theory and enable also a consistent generalization to Cartesian impedance control.

Moreover, by appropriate scaling of the feedback gains, the controller structure is used to implement position, torque or impedance control. For example, high torque and torque derivative gains, zero position gain and positive velocity feedback (for compensation of viscous friction) provide a torque controller, while high position and velocity gains are used for position control, together with lower torque feedback gains for vibration damping. On the other side, the desired torque for the controller is commanded according to the expected robot dynamics (e.g., if the robot is not moving, this corresponds to the gravity torques).

\section{SUMMARY}

The DLR MIRO presented in this paper is a new versatile lightweight research robot tailored for surgical applications. The compact and slim design, realized by the design of differential couple joints and highly integrated electronics, allows close interaction of robot and surgeon directly at the operation table, where space is sparse. With its low weight of $10 \mathrm{~kg}$, the accelerated masses are reduced significantly in comparison to an industrial robot, which enhances the safety in the unstructured environment of an operating room both for the user and the patient. Despite its low weight, a maximum payload of $30 \mathrm{~N}$ and a workspace similar to those of the human arm has been achieved, opens a broad range of possible surgical applications. The integration of joint side position and torque sensors beside the common motor position sensors, enables impedance and position control on joint and Cartesian level. Based on the DLR MIRO, we are currently developing a prototype setup for endoscopic heart surgery consisting of three robot arms equipped with active endoscopic instruments and camera. This setup will be presented on the AUTOMATICA fair 2008 in Munich.

\section{ACKNOWLEDGMENTS}

The DLR MIRO robot is funded by the Bayerische Forschungsstiftung. 


\section{REFERENCES}

Green, P.S.; Hill, J.W.; Jensen, J.F.; Shah, A. (1995), “Telepresence surgery”, Engineering in Medicine and Biology Magazine, IEEE Volume 14, Issue 3, May/Jun 1995

Phee, L., Xiao, D., Yuen, J., Chan, C., F. Ho, H. Thng, C. H. Cheng, C. Ng, W. S. (2005) "Ultrasound Guided Robotic System for Transperineal Biopsy of the Prostate”, IEEE International Conference on Robotics and Automation 2005, VOL 2, pages 13151320 Publisher IEEE, ISSN: 1050-4729, USA

Albers, J., Schmidt, T., Hassfeld, S., Heid, F. and Vahl, C. (2007) "Sternotomy and craniotomy by an autonomous robot", Zeitschrift für Herz-, Thorax- und Gefäßchirurgie, Volume 21, December 2007, ISSN: 1435-1277 (Online), Publisher: Steinkopff, Germany

Albu-Schäffer, A., Ott, Ch. and Hirzinger, G. (2007) "A unified passivity based control framework for position, torque and impedance control of flexible joint robots," International Journal of Robotics Research, vol. 26, no. 1, pp. 23-39, 2007.

Haddadin, S., Albu-Schäffer, A., Hirzinger, G. (2007): "Safety Evaluation of Physical Human-Robot Interaction via Crash-Testing", Proceedings of the 2007 Robotics: Science and Systems Conference (RSS2007), Atlanta, USA, 2007

Ortmaier, T.; Weiß, H.; Hagn, U.; Nickl, M.; Albu-Schäffer, A.; Ott, C.; Jörg, S.; Konietschke, R.; LeTien, L.; Hirzinger, G. (2006): “A Hands-on-Robot for Accurate Placement of Pedicle Screws.", Proceedings of the ICRA-2006 IEEE International Conference on Robotics and Automation, pp. 4179 - 4186, ICRA 2006, Orlando, FL (USA)

Frumento, S.; Michelini, R.; Konietschke, R.; Hagn, U.; Ortmaier, T.; Hirzinger, G. (2006): “A Co-Robotic Positioning Device for Carrying Surgical End-Effectors.”, Proceedings of ESDA 2006, ESDA 2006, Torino, (I)

Sangiovanni-Vincentelli, A.; Carloni, L.; De Bernardinis, F.; Sgroi, M. (2004): "Benefits and challenges for platform-based design", Proceedings of the 41st annual ACM IEEE conference on Design automation, 2004, pp. 409 - 414, ISBN:1-58113-828-8, San Diego, CA, USA

Konietschke, R.; Ortmaier, T.; Weiss, H.; Hirzinger, G.; Engelke, R. (2004): “Manipulability and Accuracy Measures for a Medical Robot in Minimally Invasive Surgery”, Proceedings, 9th International Symposium on Advances in Robot Kinematics (ARK), Sestri Levante, Italy, June 28 - July 1, 2004

Yoshikawa, T. (1990): "Foundations of Robotics: Analysis and Control”, The MIT Press, 1990

Craig, J.J. (1986): “Introduction to Robotics: Mechanics and Control“, Addison Wesley Longman Publishing Co, 1986 
Hirzinger, G.; Sporer, N.; Albu-Schäffer, A.; Hähnle, M.; Krenn, R.; Pascucci, A.; Schedl, M. (2002): "DLR's torque-controlled light weight robot III - are we reaching the technological limits now?”, Proceedings ICRA 2002, S. 1710 - 1716, IEEE International Conference on Robotics and Automation ICRA, Washington D.C., USA, May 2002

Butterfaß, J.; Grebenstein, M.; Liu, H.; Hirzinger, G. (2001) “DLR-Hand II: Next Generation of a Dextrous Robot Hand”, Proceedings of the 2001 IEEE Int. Conference on Robotics and Automation, Seoul, Korea, 2001.

Grunwald, G.; Schreiber, G.; Albu-Schäffer, A.; Hirzinger, G. (2004): “Touch: The Intuitive Type of Human and Robot Interaction”, Grunwald, G.; Prassler, E.; Lawitzky, G.; Stopp, A.; Grunwald, G.; Hägele, M.; Dillmann, R.; Iossifidis, I.: Advances In Human Robot Interaction, Series : Springer Tracts in Advanced Robotics, Vol. 14, Springer Publishing, ISBN 3-540-23211-7

Seibold, U.; Kübler, B.; Hirzinger, G. (2005): "Prototype of Instrument for Minimally Invasive Surgery with 6-Axis Force Sensing Capability”, ICRA 2005 IEEE International Conference on Robotics and Automation, CD ICRA 2005, S. 498 - 503, Barcelona, Spain, April 18-22, 2005

IC-Haus (2003): “BiSS Interface, Protocol Description, Release B2”, IC-Haus (http://www.BiSS-ic.de/)

Quang N., Dittrich J., “Praxis der feldorientierten Drehstromantriebsregelungen”, Publisher: Expert Germany, 1999

Benveniste, A., Caspi, P., Edwards, S., Halbwachs, N., Le Guernic, P, de Simone, R. (2003): “The synchronous languages 12 years later", Proceedings of the IEEE, vol. 91, pp. 64-83, 2003

Jörg, S., Nickl, M., Hirzinger, G. (2006): "Flexible signal-oriented hardware abstraction for rapid prototyping of robotic systems", Proceedings of the .International Conference on Intelligent Robots and Systems, Beijing, 2006

ECSS European Cooperation for Space Standardization (2003) "ECSS E-50-12A SpaceWire - Links, nodes, routers and networks"

Ott, Ch., Albu-Schäffer, A. and Hirzinger, G. (2004), “A passivity based Cartesian impedance controller for flexible joint robots - Part I: Torque feedback and gravity compensation", in IEEE International Conference on Robotics and Automation, 2004, pp. 26592665.

Le-Tien, L., Albu-Schäffer, A., Hirzinger, G. (2007) "MIMO State Feedback Controller for a Flexible Joint Robot with Strong Joint Coupling", IEEE International Conference on Robotics and Automation, April, 2007, Rome, Italy 